\title{
Factors Contributing to Preferential Motor Reinnervation in the Primate Peripheral Nervous System
}

\author{
Roger D. Madison, ${ }^{1,2,3}$ Simon J. Archibald,, ${ }^{1}$ Robert Lacin, ${ }^{1}$ and Chris Krarup ${ }^{4}$ \\ ${ }^{1}$ Division of Neurosurgery and ${ }^{2}$ Department of Neurobiology, Duke University Medical Center, and ${ }^{3}$ Research Service of \\ the Veterans Affairs Medical Center, Durham, North Carolina 27710, and ${ }^{4}$ Department of Clinical Neurophysiology, \\ University Hospital, Copenhagen, Denmark
}

Functional recovery after nerve lesions in the peripheral nervous system requires the accurate regeneration of axons to their original target end organs. This paper examines axonal regeneration of the primate median nerve lesioned at the wrist over nerve gap distances of up to $50 \mathrm{~mm}$. Nerve gaps were bridged by either a sural nerve graft or a biodegradable collagen nerve guide tube, and recovery was followed for up to 1100 d. Nondestructive physiological methods were used to serially examine the number of regenerated motor units, and binomial statistics were used to compare the observed number of regenerated motor units with that expected if axonal regeneration of motor neurons were random. We found up to twice the number of motor units expected by random regeneration in direct suture and sural cable graft groups but not in nerve guide repairs of 20 or $50 \mathrm{~mm}$. In all repaired nerves, aberrant motor axon collaterals were detected in digital sensory nerve territory. The results support the contention that the aberrant fibers represent collaterals of an $\alpha$-motor axon, which also innervates muscle. Although the aberrant motor axon collaterals remained in digital sensory nerve territory for long periods, they remained relatively immature compared with their sibling collateral projecting to muscle, or sensory axons within the digital nerve. The number of such aberrant motor axon collaterals decreased over time in some repair groups, suggesting a selective pruning of the inappropriate collateral under certain conditions.

Key words: preferential motor reinnervation; regeneration accuracy; nonhuman primate; median nerve; PNS; nerve regeneration
The major determinant of functional recovery after nerve lesions in the peripheral nervous system is the accurate regeneration of axons to their original target end organs. The definitive measure of such accuracy is physiological proof of functional reinnervation of the distal end organ target. Regenerating axons exhibit a strong preference to grow along Schwann cell basal lamina tubes in the distal nerve stump, i.e., "bands of Büngner" (Büngner, 1891; Cajal, 1928; Holmes, Young, 1942; Ide, 1983; Scherer and Easter, 1984). Myelinating Schwann cells in the normal nerve are associated with just a single axon and form continuous, unbranched basal lamina tubes all the way from a nerve transection site to the distal end organ target. As long as these basal lamina tubes remain intact they will forcibly direct regenerating axons into the previous terminal nerve branches and will thus determine the ultimate destination of the regenerating axons (Gutmann and Sanders, 1943; Brown and Hopkins, 1981; Brown and Hardman, 1987; Lee and Farel, 1988). The generation of several collateral branches from lesioned axons is one mechanism by which regeneration accuracy is increased. Increasing the number of collaterals per axon at the nerve transection site increases the chance that

\footnotetext{
Received May 3, 1999; revised Sept. 20, 1999; accepted Sept. 30, 1999.

This study was supported by National Institutes of Health Grant NS22404-13 (R.D.M.), the Medical Research Service and the Rehabilitation Research and Development Service of the US Department of Veterans Affairs (R.D.M.), Integra LifeSciences Inc. (R.D.M.), and the Danish Medical Research Council (C.K.). We thank Lisa Wrage for excellent technical assistance.

Correspondence should be addressed to Dr. Roger D. Madison, Division of Neurosurgery, Duke University Medical Center, Durham, NC 27710. E-mail: madis001@mc.duke.edu.

Dr. Archibald's present address: Integra LifeSciences, Plainsboro, NJ 08536.

Dr. Lacin's present address: Goldsboro Neurological Surgery, Goldsboro, NC 27534.

Copyright (C) 1999 Society for Neuroscience $0270-6474 / 99 / 1911007-10 \$ 05.00 / 0$
}

at least one collateral will enter a band of Büngner, which will guide it back to its original innervation target.

Preferential motor reinnervation (PMR) refers to the proven ability of regenerating motor axons in rat femoral nerve to preferentially, albeit incompletely, reinnervate muscle versus cutaneous distal nerve branches. Previous work with the rat femoral nerve has revealed that given equal access, regenerating motor axons preferentially reinnervate the terminal muscle branch, i.e., PMR (Brushart, 1988; Madison et al., 1996). Regenerating motor axons initially grow into both nerve branches but over time are pruned from the cutaneous branch (Brushart, 1990). This suggests that immediate mechanical guidance is unlikely to be crucial, and that over time the muscle branch becomes a preferred environment for motor axons because of influences from both the pathway itself as well as end organ reinnervation (Brushart et al., 1998).

Former studies describing PMR have been limited to quantifying regeneration accuracy at the terminal nerve branch level in rodents by using retrograde tracing methods. Correct choices at the nerve branch level are necessary for functional regeneration but may not be sufficient. Just because an axon has chosen the correct nerve branch does not mean that it will be able to reinnervate the correct distal receptor. The current study addressed the following questions: (1) can the functional reinnervation of target muscle, as judged physiologically, be used as an index of PMR; (2) is PMR limited to lower vertebrates, or can it be demonstrated in nonhuman primates; and (3) does the type of nerve repair procedure (e.g., autograft vs nerve guide tube) affect PMR?

As a model system, we used the median nerve of nonhuman primates, which at the wrist level divides into distinct muscle and cutaneous branches. Motor axons innervate the thenar muscles, 
whereas sensory cutaneous axons form the digital nerves of digits I-III and the radial half of digit IV. After median nerve transection and repair, we performed "motor unit counting" to determine the likelihood that regenerating motor neurons were reinnervating distal muscle at levels greater than that expected by random regeneration. These studies address the general question of motor neuron regeneration accuracy at the terminal nerve branch level (i.e., muscle vs cutaneous) and their eventual functional connection to muscle, rather than the possibility of specific motor neurons reinnervating specific muscles.

The results of these experiments are the first to show that PMR takes place in the nonhuman primate and expand our understanding of PMR by quantifying the degree to which regenerating motor axons functionally reinnervate their correct distal end organ, i.e., muscle. The extent of PMR was dependent on the type of nerve repair, and although pruning of inappropriate motor axon collaterals from sensory branches can contribute to eventual regeneration specificity, aberrant collaterals can also remain in sensory nerve for extended periods. Understanding the limits of PMR in nonhuman primates will be helpful in designing the most effective nerve repair procedures for humans.

\section{MATERIALS AND METHODS}

Surgery. Forty-one median nerve transections and repairs were performed in Macaca fasicularis adult male monkeys. The use of nonhuman primates for these studies was justified by the need to relate the time course and extent of physiological recovery to human nerve repair procedures. One of the most common sites of peripheral nerve injury in humans involves lesions to the median nerve immediately above the wrist. In addition, some of the materials used in these studies are being evaluated for eventual human use; thus it was necessary to use an animal model that closely simulates the human clinical situation.

All surgical, electrophysiological, and terminal procedures were performed under deep general anesthesia with ketamine $(12.0 \mathrm{mg} / \mathrm{kg})$ and acepromazine maleate $(1.0 \mathrm{mg} / \mathrm{kg})$. Median nerve transection at the wrist results in sensory loss of the palmar surface and finger tips of digits I, II, and III and the radial aspect of the palmar surface of digit IV. However, radial and ulnar innervation provide protective sensation to the dorsal surface of the hand and digits. Despite these sensory and related motor deficits the animals could easily grasp the cage bars, and normal behaviors such as feeding and grooming were not significantly affected. Surgical procedures, electrophysiological assessments, animal housing, and enrichment programs were approved by the Duke University Animal Care and Use Committee. Animals were monitored by the veterinary staff on a daily basis, and no animal displayed distress or any secondary complications resulting from the median nerve lesions such as self-mutilation, pressure ulcers, sensory neglect, or infection.

For the direct suture ( $n=4$ nerves) and $5 \mathrm{~mm}$ gap groups $(n=5$ nerves each autograft or collagen nerve guide) the median nerve was transected $2 \mathrm{~cm}$ above the wrist, and a $5 \mathrm{~mm}$ section of nerve was removed. The nerve was then mobilized within the muscle bed for $\sim 5 \mathrm{~cm}$ to decrease potential tension at the repair site. In the direct suture group the nerve stumps were aligned and joined by three epineurial sutures placed equidistant around the circumference of the nerve. In the autograft group, the nerve segment was removed, reversed, rotated $180^{\circ}$, and then re-implanted between the nerve stumps using epineural sutures. In the contralateral wrist, the $5 \mathrm{~mm}$ nerve gap was bridged with a collagen nerve guide using single 10-0 sutures to secure each nerve stump within the nerve guide.

For the $20 \mathrm{~mm}(n=16$ nerves $)$ and $50 \mathrm{~mm}(n=11$ nerves $)$ repair groups $\sim 1.5$ and $4 \mathrm{~cm}$ of median nerve were removed, respectively, beginning $2 \mathrm{~cm}$ above the wrist and continuing proximally toward the elbow. Standard sural cable grafts consisting of 20 or $50 \mathrm{~mm}$ portions of sural nerve were used to bridge eight of the $20 \mathrm{~mm}$ and six of the $50 \mathrm{~mm}$ nerve repairs, respectively. The remaining eight of the $20 \mathrm{~mm}$ and five of the $50 \mathrm{~mm}$ nerve repairs were performed with collagen nerve guides as described above. The construction and physical properties of the type I collagen nerve guides used in this study have been described previously (Li et al., 1992; Archibald et al., 1995; Madison and Archibald, 1996).

Standard electrophysiology. Beginning at weekly and then monthly intervals, nondestructive physiological assessments were performed, which covered a period of up to $1100 \mathrm{~d}$ after nerve repair. During each physiological session, both arms of the animal were examined. A total of more than 1000 physiological sessions (approximately split between the various repair groups) were performed.

Motor conduction studies. The median nerve was electrically stimulated at the wrist at the level of the nerve lesion by rectangular pulses with a duration of $0.1-0.2 \mathrm{msec}$ using a constant current stimulator and insulated steel needles $(0.7 \mathrm{~mm}$ in diameter with a $3 \mathrm{~mm}$ bared tip). Evoked compound muscle action potentials (CMAPs) were recorded from the abductor pollicis brevis muscle (APB) using a subcutaneous needle placed over the motor end plate zone of the muscle (see Fig. $4 A$ for electrode placements). The CMAP was amplified $(10 \mathrm{~Hz}-10 \mathrm{kHz}, 3 \mathrm{~dB}$ down) and recorded digitally; amplitudes were measured peak to peak; and latencies were measured to the first deflection from the baseline. Optimal placement of the stimulating electrode close to the nerve was assessed by obtaining a threshold of activation of the CMAP of $\leq 0.6$ $\mathrm{mA}$. Once optimally situated, this electrode was kept in place during the remainder of the physiological session to subsequently record evoked sensory potentials (see below). The anode was placed subcutaneously at a transverse distance of $10-20 \mathrm{~mm}$ from the wrist stimulating electrode, and the stimulus current was increased above that which elicited a maximal CMAP. During early regeneration the threshold of nerve fibers is markedly elevated, and at this stage a maximal stimulus of $60 \mathrm{~mA}$ and a duration of $1 \mathrm{msec}$ was used to ascertain whether the muscle had become reinnervated. After maturation of the regenerated fibers, more standard stimulus intensities (e.g., $<3-8 \mathrm{~mA}$ ) were adequate to evoke a maximal CMAP response.

Sensory conduction studies. Sensory nerve fibers in digit II were stimulated with needle electrodes at the base and tip of the digit, or a surface ring electrode was placed at the midlevel of the proximal phalanx (see Fig. $4 A$ for electrode placements). The evoked compound sensory action potentials (CSAPs) were recorded from the needle electrode that had been optimally situated close to the median nerve at the wrist to evoke the CMAPs (see above). In selected sessions, this recording electrode was also used to ascertain sensory potentials arising from reinnervated mechanoreceptors evoked by a pulsatile tactile stimulator applied to the distal finger pad or proximal phalanx of digit II (for procedural details see Archibald et al., 1995). When the digit was stimulated with needle electrodes at both the tip and base of the digit, the differences in the recorded latencies at the wrist was used to calculate the sensory nerve conduction velocity (SNCV) between the two stimulation sites in digit II. All evoked sensory responses were amplified $(200 \mathrm{~Hz}-4 \mathrm{kHz}, 3 \mathrm{~dB}$ down), averaged (sampling interval, $20-40 \mu \mathrm{sec}$ ), and digitally recorded. The CSAP amplitude was measured peak to peak, and the latency at the wrist was measured to the first positive peak of the potential.

Aberrant motor responses evoked from stimulating digit II. In all of the regenerated nerves (but never in normal nerve) a response could be evoked in the APB by stimulating the sensory nerve territory of digit II. Evidence is presented below that these recurrent responses arise from aberrant motor axon collaterals that have inappropriately grown into the sensory nerve territory in digit II in addition to the main axon, which innervates muscle. We have therefore termed such aberrant responses recurrent CMAPs (recCMAPs).

recCMAPs were recorded from the APB after electrical stimulation of digit II as described above for the sensory conduction studies. The high stimulus threshold of fibers giving rise to the recCMAPs during early regeneration required stimulus currents of up to 50-70 $\mathrm{mA}$ to determine whether a response was present. After maturation of the regenerated fibers lower stimulus intensities were required (e.g., 10-15 mA); however, they remained above the stimulus intensities necessary to activate the direct CMAP or the CSAP. When digit II was stimulated both at the tip and base of the digit, the difference in the recCMAP latencies was used to calculate the conduction velocities of the aberrant motor axon collaterals present between the two stimulation sites in digit II.

Motor unit number estimation. Motor unit number estimation (MUNE) was used for the serial counting of the number of motor units in the median nerve innervated hand muscles (Brown, 1972; Sica et al., 1974; Fu and Gordon, 1995a,b). This nondestructive physiological technique detects distinct incremental increases in the amplitude of the evoked CMAP as the stimulus current is gradually increased from threshold level. Each distinct increase in the CMAP represents the recruitment of a single additional motor unit (see Fig. 1). Stimuli were first delivered at or slightly above the threshold needed to evoke the smallest CMAP. Stimulus intensity was then carefully increased to evoke discrete incre- 
ments in the CMAP. These "jumps" in the CMAP were followed on a storage oscilloscope and reflect the combined contributions of successively recruited individual motor units. The total number of motor units was then estimated by dividing the amplitude of the maximally evoked CMAP by the average amplitude of the first $(\sim 5-15)$ discrete motor unit potentials. In this manner, the number of evoked motor units from median nerve reinnervated muscles was followed serially from $\sim 400$ $1100 \mathrm{~d}$ postoperatively. Systematic MUNE was not initiated until after the killing of the $5 \mathrm{~mm}$ group, and the limited data regarding the number of motor units for this group were not included in the analysis.

A well known limitation of the technique of MUNE is that the total number of motor units must be estimated from just the first few discrete increments of the CMAP. It is thus reassuring that the baseline estimates obtained using this technique in a serial nondestructive manner agreed with an estimate obtained after extensive dissection of the hand and stimulation of individual nerve branches under visual guidance (see below). In addition, in most of the recording sessions for reinnervated muscle virtually all of the motor units could be discerned as distinct jumps in the evoked CMAPs, and extrapolation using just the first few distinct CMAPs was not necessary.

To verify the origin of the muscles giving rise to the recorded evoked CMAP, a terminal recording session was performed on one unoperated hand. Extensive dissection was performed to visually identify each of the median nerve innervated thenar and lumbrical muscles and their respective terminal nerve branches. Under visual guidance, a recording electrode was serially inserted into each of the identified muscles, and the median nerve was stimulated at the wrist and then at the terminal nerve branch for that particular muscle. After recording of the number of evoked motor units at each muscle, the terminal nerve branch to that individual muscle was cut. The complete dropout of the CMAP after stimulation at the wrist or terminal nerve verified that the previous potentials were arising exclusively from that particular muscle. The very close agreement between estimates of the number of motor units during the one terminal recording session and the more extensively applied nondestructive closed hand procedures (147 and 155; see Table 1), shows that the large surface area of the recording electrodes used during the nondestructive sessions recorded motor units from all of the median nerve innervated hand muscles in these very small hands. There was thus no detectable bias in terms of the placement of the recording electrode and the source epicenter, as can be demonstrated when recording from much larger extremity muscles.

Statistical estimation of the "expected" number of regenerated motor units and defining the null hypothesis of random regeneration. We estimated the number of myelinated axons at the wrist level that eventually project to the median nerve innervated hand muscles to determine the number of hard-wired basal lamina pathways at the median nerve transection site, which would direct an axon to muscle. Standard quantitative morphometrics were performed as previously described (Archibald et al., 1995). Total numbers of myelinated axons were quantified from electronmicroscopic images at a final magnification of $6000 \times$.

MUNE was used to follow the number of regenerated motor units. It is important to stress that the method of MUNE is specific for $\alpha$-motor neurons, because neither sensory axons that grow into muscle nor regenerating $\gamma$ motor neurons (normally innervating intrafusal muscle fibers) are able to generate evoked CMAPs (Weiss and Edds, 1945; Gutmann, 1945; Zalewski, 1970; Scott, 1987). The probability of an $\alpha$-motor axon being directed to the median nerve innervated hand muscles can thus be defined as the number of myelinated Schwann cell tubes to the median nerve innervated hand muscles divided by the total number of myelinated Schwann cell tubes at the transection site. Thus, if we assume that a regenerating $\alpha$-motor axon is a random variable $X$, then in terms of "correctly" reaching the median nerve innervated hand muscles it will have a binomial distribution $(X \cap B)$ with parameters $n$ and $p$ (Zar, 1984 ), where $n$ is the number of regenerating $\alpha$-motor neurons, and $p$ is the probability of making the "correct choice" to the median nerve innervated hand muscles (i.e., number of myelinated Schwann cell tubes to the median nerve innervated hand muscles/total number of myelinated Schwann cell tubes at the transection site). This expected probability is then defined as: $E(X)=n p$, with $\operatorname{SD}(X)=\sqrt{n p(1-p)}$. To relate the observed number of regenerated motor units $(y)$ with the predicted chance level $(X), X$ is converted to a $Z$ score, and one determines the probability of the observed $y$ being different from the predicted level of $X$ by $P(X>y) \sim P(Z>[y-E(X) / \operatorname{SD}(X)]$, where $Z$ is the standard normal score (Zar, 1984).

Studies to elucidate the anatomical basis of the recCMAP. In several recording sessions, $2 \%$ lidocaine was injected at the base of digit II to determine whether the high stimulus strength initially used to excite the aberrant motor fibers $(50-70 \mathrm{~mA})$ could spread to activate nonmedian nerve pathways (e.g., ulnar nerve). In other sessions, $2 \%$ lidocaine was injected into the forearm or upper arm to block nerve conduction above the lesion site. To investigate the possibility of ephaptic transmission, we studied the effect on recCMAP potentials of cooling, ischemia, and refracted doses of local intravenous lidocaine.

\section{RESULTS}

\section{Morphological characteristics of the median nerve and the expected number of regenerated motor units}

As noted above, to conclude whether regenerating motor neurons reinnervate muscle at levels greater than that expected by random regeneration, it is necessary to estimate the number of myelinated Schwann cell tubes at the cut nerve face, which would direct a regenerating axon to the previously innervated muscles. Standard morphometric techniques revealed $8449 \pm 583$ myelinated axons at the wrist level for normal median nerve $(n=4$, mean \pm SEM). Although we considered directly counting the number of myelinated axons in the terminal muscle branches, it has previously been shown that these axons display extensive branching before the discrete anatomical formation of the terminal branches themselves (Wray, 1969; R. D. Madison, S. J. Archibald, and C. Krarup, unpublished observations). Thus it is impossible to obtain reliable unbranched axon estimates at this level by morphometrics alone.

By contrast, the number of $\alpha$-motor neurons can be estimated by MUNE and in the unlesioned median nerve was found to be $147 \pm 14$ (this paper; see below). Based on axon diameter and conduction velocity measurements, previous studies have estimated $\gamma$ motor neurons to make up between 19 and $45 \%$ of a total motor neuron pool (McLeod and Wray, 1967; Henneman, 1974; Swett et al., 1986). To be conservative, if one assumes the $45 \%$ level, it would add $120 \gamma$ motor neurons to the $147 \alpha$-motor neurons to bring the total motor neuron pool to these muscles to 267.

Previous denervation studies in the primate have estimated that the number of myelinated sensory fibers to the intrinsic hand muscles is approximately equal to or less than the number of myelinated motor fibers (McLeod and Wray, 1967; Wray, 1969). Thus doubling the number of myelinated axons from motor axons would bring the total number of myelinated axons in the terminal muscle branches to 534 . By dividing the number of myelinated axons at the cut nerve face (8449) by the number of myelinated axons eventually projecting to the terminal muscle branches (534), one can estimate the proportion of continuous Schwann cell tubes at the cut face that project directly to terminal muscles (0.06). This resulting fraction was used to multiply the normal number of $\alpha$-motor units $(0.06 \times 147)$ to estimate the average number of $\alpha$-motor units expected to return to these muscles after random axon regeneration: 8.82. Using binomial statistics, an SD can be placed around this average, $8.82 \pm 2.9$ (see above; Zar, 1984).

\section{Physiological characteristics of motor units at $>900 \mathrm{~d}$ after repair and comparison with the expected number of motor units}

In an attempt to compare stabilized motor units, we analyzed the number of motor units in the direct CMAP and the recCMAP at time periods of $>900$ d (i.e., $\sim 2.5$ years after surgery). We calculated $147 \pm 14$ (mean \pm SEM) motor units in the normal median nerve innervated hand muscles ( $n=16$ nerves, 64 independent recording sessions). A single terminal recording session after extensive dissection of the hand revealed 155 distinct motor 


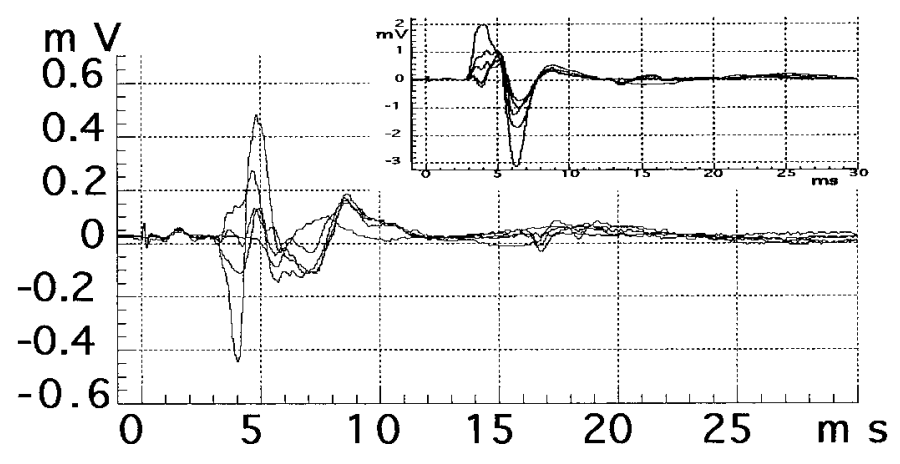

Figure 1. Traces from a physiological MUNE session in a muscle reinnervated by regenerating median nerve motor axons (data are from a nerve $1046 \mathrm{~d}$ after repair with a $20 \mathrm{~mm}$ sural nerve graft). A total of 15 motor units were observed during this recording session, but for clarity only a few are illustrated. Main figure, Initial motor units evoked by stimulating the median nerve at the wrist using currents at and just above the threshold level for a detectable CMAP $(\sim 0.1 \mathrm{~mA})$. Inset, Recruitment of the last few motor units including the maximal CMAP $(\sim 2 \mathrm{mV})$ evoked by a supramaximal stimulus.

units in the median nerve innervated hand muscles. This indicates that during the nondestructive serial physiology sessions the pick-up area of the subcutaneous needle electrode was wide enough to record motor units from all of the hand muscles innervated by the median nerve (i.e., thenar and lumbricals). Results of a physiological assessment for the number of motor units in reinnervated muscle are shown in Figure 1.

The number of motor units for the CMAP in the various nerve repair groups at time points $>900 \mathrm{~d}$ is listed in Table 1. Also shown in Table 1 is the statistical comparison with the expected number of motor units if motor axon regeneration were random, as predicted by binomial probability statistics (Zar, 1984; see Materials and Methods). The direct suture and both sural cable graft groups (20 and $50 \mathrm{~mm}$ ) displayed significantly more motor units than expected. Neither of the nerve guide repair groups (20 and $50 \mathrm{~mm}$ ) was significantly different from the expected number of motor units.

At these late time points, the average amplitude of the motor units in the CMAP after nerve regeneration was significantly larger for all repair groups compared with the normal size of $0.2 \pm 0.1 \mathrm{mV}$ (range, $0.7-1.5 \mathrm{mV} ; p<0.05$; Table 1). The number of motor units and their sizes in the recCMAP were also assessed using the same procedures. Within each repair group at these late time points the average motor unit amplitude of the recCMAP was significantly smaller than that of the CMAP (range, 0.4-0.9 $\mathrm{mV} ; p<0.001$; Table 1).

There was a significant negative correlation between the average amplitude of the final motor units ( $>900 \mathrm{~d})$ making up the CMAP and the time to the first detectable CMAP (Fig. 2). There was also significant variability among the groups in terms of the amount of time from the initial surgery to the first detectable CMAP (Fig. 3).

\section{Anatomical basis for the recCMAP}

When $2 \%$ lidocaine was injected into the base of digit II, both the CSAP and the recCMAP evoked by stimulation at the digit tip was abolished, whereas the CMAP evoked by stimulation at the wrist was not affected, thereby ruling out stimulus spread as a basis for the recCMAP (data not shown). To exclude the possibility that the recCMAP might be attributable to reflex activity, the median nerve was blocked in the forearm or upper arm by local injection of lidocaine. A hollow needle with a $3 \mathrm{~mm}$ bared tip was placed close to the median nerve (as indicated by a low CMAP threshold when used for stimulation) and then used to inject $2 \%$ lidocaine. The local anesthesia caused block of conduction along the nerve in the forearm but had no effect on the recCMAP elicited by digit II stimulation (Fig. 4B), thus excluding the possibility that the recCMAP represented a CNS reflex.

To test whether the recCMAP represented dispersed compound motor activity evoked by stimulation of the digital nerve or whether a single fiber or a few fibers carried the afferent activity, the effect of double stimulation was evaluated. The recCMAP shown in Figure $4 B$ consisted of three different spikes (Pot 1-3), which were evoked at twice the stimulus intensity that elicited a maximal CSAP (Fig. 4C). To record the responses (R1 and R2) to the two stimuli ( $\mathrm{S} 1$ and $\mathrm{S} 2$ ), the responses $\mathrm{R} 1+\mathrm{R} 2$ were recorded digitally, and the response $\mathrm{R} 1$ to $\mathrm{S} 1$ alone was then electronically subtracted. The three components of the recCMAP had different absolute refractory periods of 1.3, 2.0, and $1.45 \mathrm{msec}$, respectively (Fig. 4D). The full amplitudes of the individual spikes were reached at the absolute refractory period, and at longer time intervals there was no further change in amplitude (Fig. $4 F$ ). In contrast, the CMAP evoked by stimulation of the median nerve at the wrist had an absolute refractory period of $0.9 \mathrm{msec}$, which was $\sim 30 \%$ shorter than the shortest refractory period for the recCMAP (Fig. 4E). Moreover, the amplitude of the CMAP gradually increased (Fig. $4 F$ ) during the relative refractory period at longer $\mathrm{S} 1-\mathrm{S} 2$ intervals, as expected from a response that was the sum of several motor units. The abrupt rise of the recCMAP to its maximal amplitude at the absolute refractory period suggests that only a few digital nerve fibers with all-or-nothing behavior contributed to the afferent pathway along which the activity giving rise to the recCMAP was propagated. When recorded at threshold the recCMAP in some instances also suggested an all-or-nothing behavior. The latencies of both the recCMAP and the CMAP gradually decreased during the relative refractory period (data not shown).

Stimulation of mechanoreceptors using a pulsatile tactile stimulator applied to the distal pad or proximal phalanx of digit II resulted in a sensory potential that could be recorded at the wrist; however, even after averaging up to 1000 traces no activity was detected in muscle (data not shown).

The effect of cooling and local intravenous lidocaine on the recCMAP was examined to explore the possibility of ephaptic transmission (data not shown). Ephaptic transmission usually occurs from unmyelinated fibers requiring higher stimulus intensities to larger myelinated fibers with lower stimulus thresholds. Ephaptic responses are considered to have a lower safety margin of transmission compared with direct electrical conduction, and thus their occurrence can be increased by prolonging the amount of time that action potentials traverse a repair site (Rasminsky, 1980). When the skin temperature of the hand and forearm was reduced from 35 to $20^{\circ} \mathrm{C}$ by packing the arm in ice, the latency of the CMAP increased by $40 \%$, and the amplitude decreased by $25 \%$. The latency of the recCMAP increased by $65-70 \%$, whereas the amplitude did not change systematically. No further recCMAP components were recruited at low temperature.

Lidocaine ( $10 \mathrm{ml}$ of $0.02 \%$ ) was injected intravenously distal to a blood pressure cuff. Within $10 \mathrm{~min}$, the CMAP and recCMAP were each reduced by $\sim 20 \%$. The cuff was released, and the recCMAP followed until it returned to baseline. Ischemia was again induced, and $10 \mathrm{ml}$ of $0.2 \%$ lidocaine was injected. Within $10 \mathrm{~min}$, the CMAP and recCMAP were each reduced by $\sim 50 \%$. 
Table 1. Characteristics of motor units in the direct (CMAP) and the recurrent motor response (recCMAP) in regenerated median nerves at times $>900$ d after repair

\begin{tabular}{|c|c|c|c|c|c|}
\hline \multirow[b]{2}{*}{ Procedure } & \multicolumn{3}{|l|}{ Motor unit no. } & \multicolumn{2}{|c|}{ Motor unit amplitude (mV) } \\
\hline & $\begin{array}{l}\text { CMAP: no. of } \\
\text { motor units } \\
(\text { mean } \pm \mathrm{SD})\end{array}$ & $\begin{array}{l}\text { No. of motor units } \\
\text { in CMAP vs } \\
\text { expected }\end{array}$ & $\begin{array}{l}\text { recCMAP: no. of } \\
\text { motor units } \\
(\text { mean } \pm \text { SEM })\end{array}$ & $\begin{array}{l}\mathrm{CMAP}^{*} \\
(\text { mean } \pm \text { SEM) }\end{array}$ & $\begin{array}{l}\text { recCMAP** } \\
(\text { mean } \pm \text { SEM })\end{array}$ \\
\hline Normal, closed hand $(n=64 ; 16)$ & $147 \pm 14$ & & & $0.2 \pm 0.1$ & \\
\hline Normal, open surgery $(n=1)$ & 155 & & & $0.17 \pm 0.05$ & \\
\hline Direct suture $(n=19 ; 4)$ & $18.4 \pm 3.2$ & $p<0.001$ & $2.7 \pm 0.5$ & $1.5 \pm 0.2$ & $0.9 \pm 0.3$ \\
\hline Graft, $20 \mathrm{~mm}(n=29 ; 8)$ & $14.9 \pm 1.9$ & $p<0.02$ & $3.1 \pm 0.3$ & $1.3 \pm 0.1$ & $0.5 \pm 0.1$ \\
\hline Graft, $50 \mathrm{~mm}(n=25 ; 6)$ & $16.3 \pm 2.9$ & $p<0.01$ & $3.5 \pm 0.5$ & $0.7 \pm 0.1$ & $0.4 \pm 0.1$ \\
\hline Tube, $20 \mathrm{~mm}(n=38 ; 8)$ & $12.5 \pm 1.9$ & NS & $2.3 \pm 0.4$ & $1.2 \pm 0.1$ & $0.9 \pm 0.3$ \\
\hline Tube, $50 \mathrm{~mm}(n=15 ; 3)$ & $9.7 \pm 2$ & NS & $1.7 \pm 0.5$ & $0.9 \pm 0.1$ & $0.7 \pm 0.1$ \\
\hline Expected & $8.8 \pm 2.9$ & & & & \\
\hline
\end{tabular}

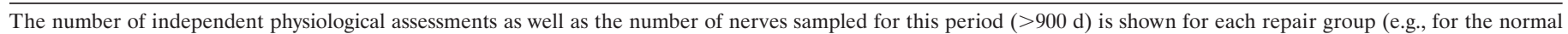

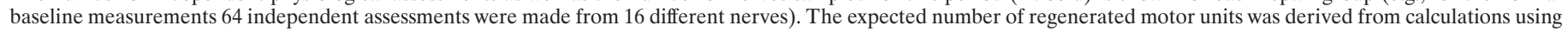
binomial statistics to model "random" motor axon regeneration (see Results and Discussion for details).

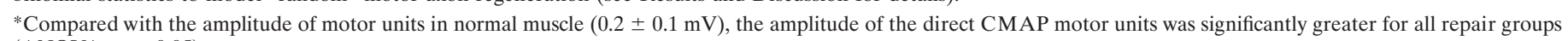
(ANOVA, $p<0.05)$.

**Within each of the repair groups, the motor unit amplitude of recCMAPs was significantly smaller than that of the direct CMAP ( $p<0.001$, paired $t$ tests).

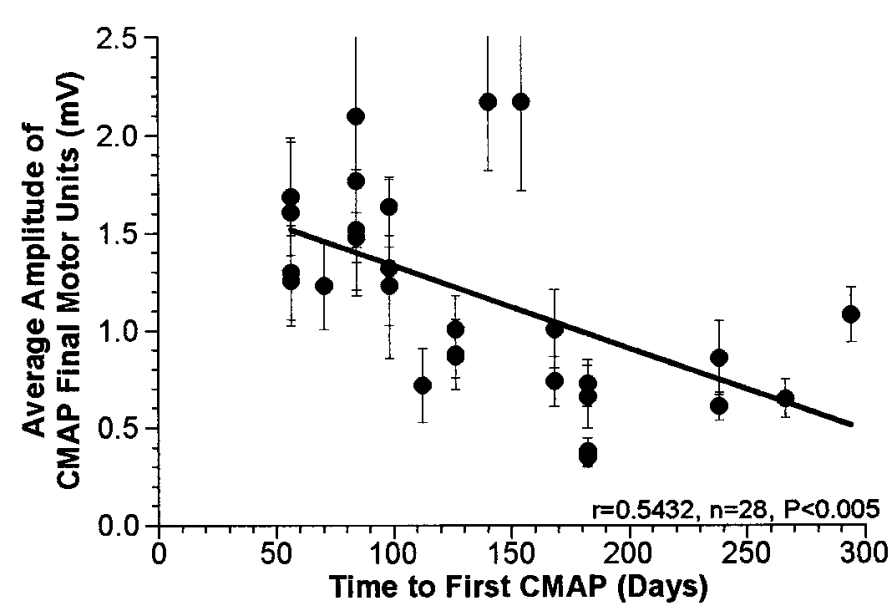

Figure 2. Correlation between average amplitude of the motor units in the CMAP at $>900 \mathrm{~d}$ and the initial time delay until the first detectable CMAP (two-tailed test). Each repaired nerve is represented by a single data point (mean $\pm \mathrm{SEM})$.

The cuff was released, and the response was allowed to recover. Ischemia was again induced, and $7.5 \mathrm{ml}$ of $0.5 \%$ lidocaine was injected. The recCMAP and CMAP were both extinguished within 3 min.

The response of the recCMAP to refracted doses of lidocaine injected intravenously suggested that it was as stable as the CMAP, rather than demonstrating a lower safety margin of transmission. The results of cooling and lidocaine injections strongly suggest the recCMAP arises from an aberrant axon collateral of an $\alpha$-motor neuron, which also sends an axon to muscle.

\section{Appearance and maturation of the recCMAP}

In the $5 \mathrm{~mm}$ repair group, the recCMAP could first be recorded $25 \mathrm{~d}$ (range, $0-42 \mathrm{~d}$ ) after a CSAP was first present ( $100 \mathrm{~d})$, and it was associated with a visible contraction of the muscle. The stimulation threshold of the recCMAP was initially much higher than that of the CSAP but gradually decreased during maturation; however, even at 350-500 d after repair, the current neces-

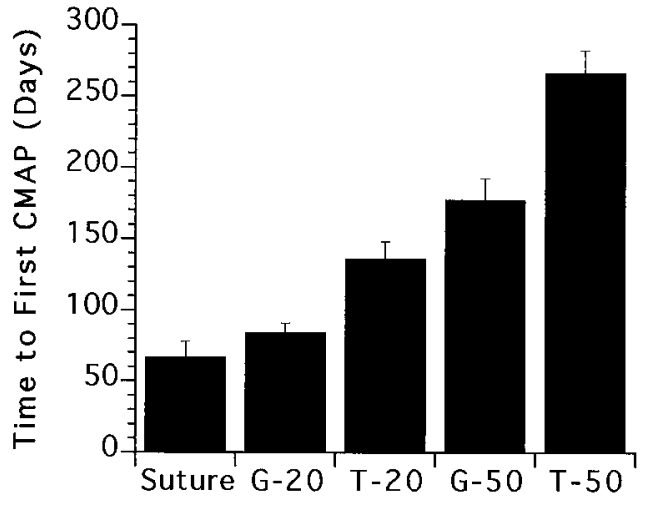

Figure 3. Comparisons of the average amount of initial time delay for each repair group until the first detectable CMAP (overall $p<0.0001$, ANOVA or Kruskal-Wallis). There was no significant difference in the delay between the suture and the $20 \mathrm{~mm}$ graft group $(G-20)$; however, both of these groups had significantly shorter delays compared with any of the remaining repair groups $(p<0.005$, Student-Newman-Keuls or Student's two tailed $t$ test, confirmed by Mann-Whitney $U$ ).

sary to evoke a maximal recCMAP remained on average $4.1 \pm$ 0.3 (mean \pm SEM) times that needed to evoke a maximal CSAP $(p<0.001$; data not shown). Similar results were found in all repair groups, although as expected, there was a correlated increase in the amount of time postoperatively until responses could be first detected depending on the initial nerve gap distance.

Throughout the observation period the latency of the recCMAP decreased exponentially but remained significantly greater than that of the CMAP or CSAP $(p<0.001$; data not shown). The conduction velocity of fibers along the same path in the digit that respectively give rise to the CSAP or the recCMAP was calculated by stimulating the digital nerve at the tip or the base of digit II (Fig. $5 A$; see Materials and Methods) and dividing this measured distance by the differences in the recorded latencies. Both conduction velocities along digit II increased gradually along S-shaped curves with similar slopes; however, the CSAP reached a higher level than the recCMAP. At 341-679 d after repair the CSAP was $34 \pm 2 \mathrm{~m} / \mathrm{sec}$, whereas the conduction 


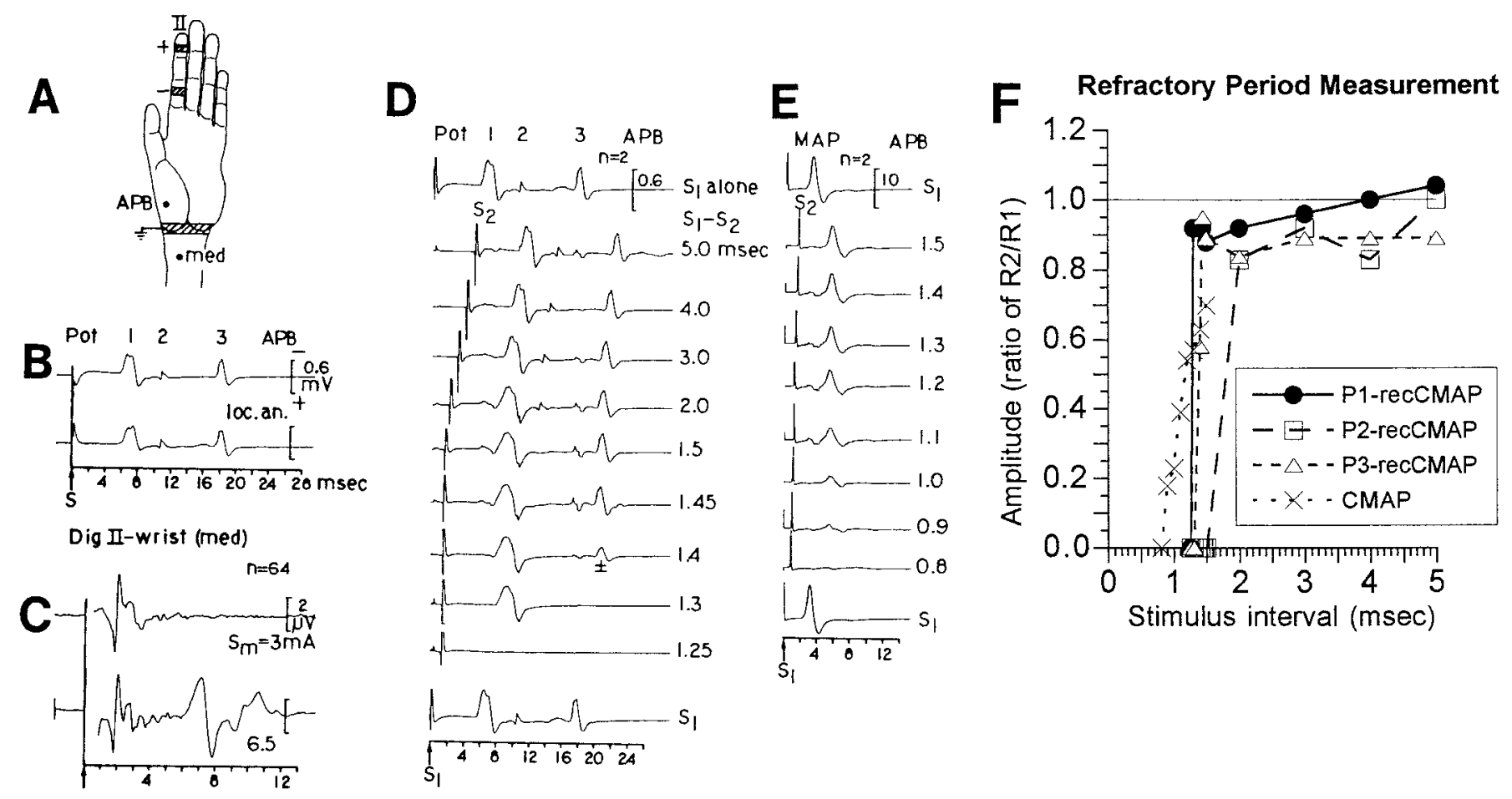

Figure 4. Recordings of CSAP, CMAP, and recCMAP in a monkey $350 \mathrm{~d}$ after median nerve repair. $A$, Monkey hand to show stimulus electrodes at the base of digit II, subcutaneous recording electrode at the APB, and electrode site for recording or stimulation of the median nerve $($ med $)$ at the wrist. $B$, recCMAP evoked by stimulation at digit II recorded at the APB before (top trace) and after (bottom trace) local anesthesia of the proximal median nerve, indicating that the recCMAP pathway was completely distal to the anesthesia application site. The individual spikes of the response are numbered Pot 1-3. C, CSAP evoked by stimulation at digit II and recorded at the wrist. The stimulus strength of $3 \mathrm{~mA}$ (top trace) was sufficient to evoke a maximal CSAP. A higher stimulus strength of $6.5 \mathrm{~mA}$ (bottom trace) evoked a far-field potential after the CSAP, which corresponded in time ( 7 msec) to Pot 1 of the recCMAP. D, Refractory period of the recCMAP. Top and bottom traces are evoked by stimulus S1 alone. The interval between S1 and S2 is indicated at the right. The response R1 to S1 was electronically subtracted from the double response R1 + R2. The respective refractory periods for Pot 1-3 were 1.3, 2.0, and $1.45 \mathrm{msec}$. At an interstimulus interval of $1.4 \mathrm{msec}$, Pot 3 was on-off showing half-amplitude in the average of two responses. E, Refractory period measurements of the CMAP indicates an absolute refractory period of $\sim 0.9$ msec, significantly shorter than any of the refractory periods observed for the recCMAPs. $F$, Amplitudes of the recCMAP and the CMAP as a ratio of the response (R2) to the test stimulus (S2) divided by the response (R1) to the conditioning stimulus (S1) alone. Note the abrupt rise of the recCMAP to its maximal amplitude at the absolute refractory period, in contrast to the graded increase displayed by the CMAP. This suggests that only a few aberrant motor axon collaterals within the sensory nerve territory with all-or-nothing behavior were responsible for the recCMAP.

velocity of the recCMAP was only $13 \pm 1 \mathrm{~m} / \mathrm{sec}$ (Fig. $5 A ; p<$ 0.001 , paired $t$ test).

The effect of ischemia was also consistent with the aberrant collaterals having a small diameter (Parry and Brown, 1982). Ischemia was induced for periods of $15-20$ min by inflating a blood pressure cuff placed around the forearm above the systolic blood pressure, and the effect on the CMAP and recCMAP was continuously monitored. The recCMAP was stable for $\sim 10 \mathrm{~min}$ and then declined rapidly and was no longer detectable by $15 \mathrm{~min}$. After $17 \mathrm{~min}$ of ischemia the amplitude of the CMAP had decreased by $10 \%$, and the latency had increased by $5 \%$. Within 2 min after deflating the cuff, the recCMAP had fully recovered.

The total conduction time in milliseconds for the recCMAP from the digit to the muscle was directly determined by its latency. The conduction time for the recCMAP was then estimated separately for time taken along the aberrant afferent portion (digit to wrist) of the pathway to the branching point at the lesion site, and the normal efferent motor portion of the pathway (i.e., wrist to muscle; Fig. $5 B$ ), as follows: the conduction time along the aberrant afferent pathway was derived by dividing the distance from the stimulation site in the digit to the wrist electrode (used for CSAP recordings or CMAP stimulation) by the recCMAP conduction velocity (see Fig. $4 A$ for electrode placements). This conduction time was significantly longer than that for the latency of the CSAP (also expressed in milliseconds) obtained by stimulating at the same site in the digit and recording directly at the same site in the wrist (Fig. 5B).

The recCMAP conduction time from the digit to the wrist was then subtracted from the total conduction time from digit to muscle (APB latency) to give the "residual" conduction time along the efferent pathway from the repair site in the wrist to the muscle. This residual conduction time of the recCMAP (in milliseconds) was not significantly different from that for the latency (in milliseconds) of the direct CMAP response evoked by stimulation at the same site in the wrist and recorded at the muscle (Fig. $5 B$ ). All of the above conduction velocity measurements were taken at $>300 \mathrm{~d}$ after repair in the $5 \mathrm{~mm}$ lesion groups, a time when the latencies had stabilized.

\section{Change in the number of motor units contributing to the CMAP and recCMAP from 400 to $1100 \mathrm{~d}$ postoperatively (Figs. 6, 7)}

In the direct suture group the correlation between time and the number of motor units contributing to the CMAP was nonsignificant, indicating that the number of motor units was not significantly changing over this period. Over the same period, however, 

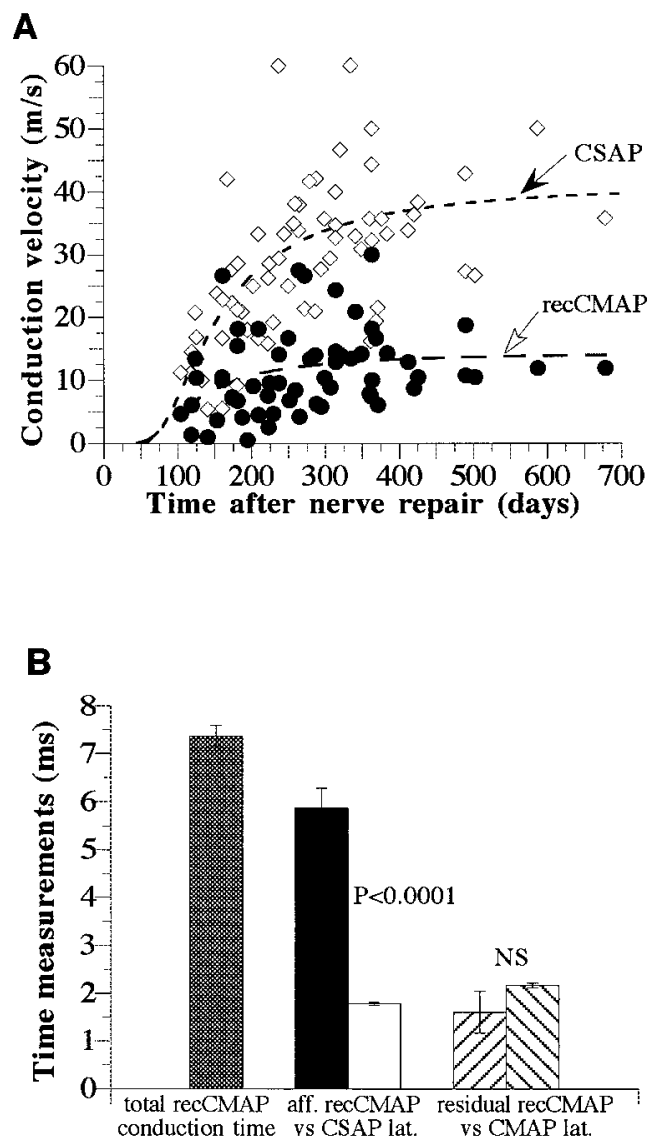

Figure 5. Conduction velocities (meters per second) and times (milliseconds) calculated from stimulating digital nerve at the tip or base of digit II. Data are from 11 median nerves (5 mm nerve lesion) and 131 physiological assessments. A, Conduction velocities of the CSAP and recCMAP along the same conduction path from the tip to the base of digit II as function of the time after nerve repair. The regression curves were calculated from the equation: $a^{*} \exp \left(b / d^{2}\right)$ using the least squares method $(r=0.4093$ and $0.7387 ; p<0.001)$. B. Estimates of conduction time, in milliseconds, of the recCMAP along the aberrant sensory (afferent) versus motor (efferent) pathways. Only time points $>300 \mathrm{~d}$ after surgery were included (see $A ; n=25$ physiological assessments). The total conduction time for the recCMAP was calculated to be $7.4 \pm 0.2 \mathrm{msec}$ (mean \pm SEM; hatched bar). The conduction time of the recCMAP along the sensory portion of the pathway (afferent; black bar), was calculated to be $5.9 \pm 0.4 \mathrm{msec}$, which is significantly longer than the latency of the CSAP (in milliseconds) over the same pathway (white bar; $1.8 \pm 0.03$ msec; $p<0.0001$, paired $t$ test). The residual conduction time of the recCMAP along the efferent motor pathway (calculated by subtraction of the afferent conduction time from the total conduction time) was not significantly different (paired $t$ test) from the measured latency (in milliseconds) of the direct motor response evoked by stimulation at the wrist.

the number of motor units contributing to the recCMAP was significantly decreasing ( $p<0.02$; Fig. 6). A similar pattern was seen in the $20 \mathrm{~mm}$ sural cable graft group, with no significant change in the number of motor units for the CMAP but a significant reduction in the recCMAP $(p<0.01$; Fig. $7 A)$. Conversely, in the $50 \mathrm{~mm}$ nerve graft group the number of motor units for the direct CMAP significantly decreased, and the number for the recCMAP significantly increased over time (Fig. $7 B$ ).

All of the nerves in the $20 \mathrm{~mm}$ nerve guide group displayed successful regeneration; however two of the nerves in the $50 \mathrm{~mm}$ nerve guide group failed to show any signs of regeneration. Thus the data for the number of motor units in the $50 \mathrm{~mm}$ nerve guide

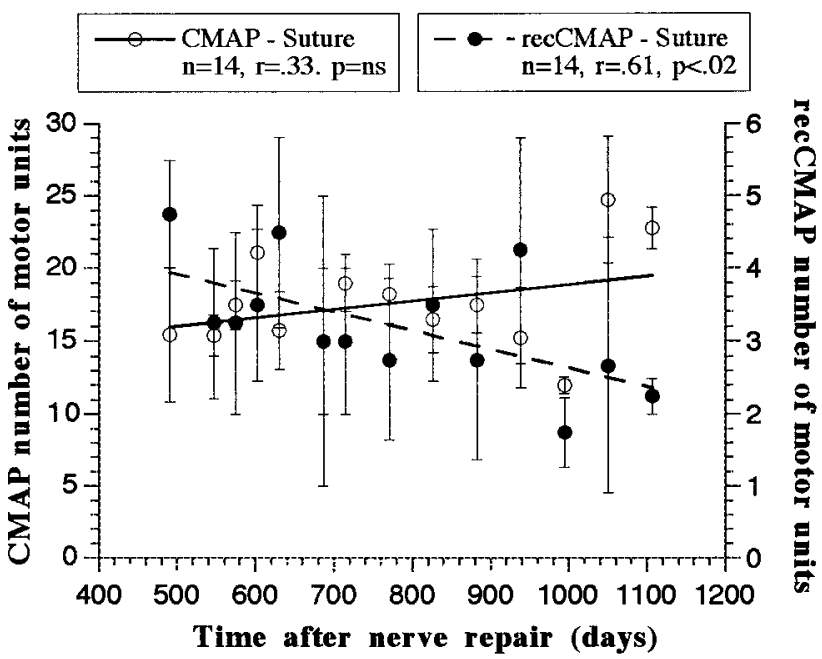

Figure 6. Number of motor units contributing to the CMAP (open circles, left $y$-axis) and the recCMAP ( filled circles, right $y$-axis) with time after nerve repair with direct suture $(n=4$ nerves). Each data point represents the mean $( \pm$ SEM) of the measurements for that time point. Linear curves were calculated from the least squares method. $n$, Number of time points used to calculate the curve; $r$, correlation between time and number of motor units; $p$, statistical significance of the correlation (twotailed test); $n s$, nonsignificant.

group are derived from the three animals that showed regeneration. For both nerve guide groups there were no significant changes in the number of motor units for either the direct or recCMAP (Fig. 7C,D).

\section{DISCUSSION}

These results suggest several major points concerning preferential motor reinnervation after primate median nerve lesions: (1) under certain circumstances regenerating motor neurons reinnervate muscle at levels that are significantly greater than expected from random regeneration; (2) there is a significant negative correlation between the initial length of time of muscle denervation and the much later final motor unit amplitudes; (3) aberrant motor axon collaterals enter cutaneous pathways and remain for extended periods, although they remain small and immature; and (4) pruning of the aberrant collateral from the cutaneous pathway over time is seen under certain conditions.

\section{Number of motor units reinnervating muscle (CMAP)}

MUNE revealed significantly more $\alpha$-motor units for the direct suture and the sural cable graft groups than expected if regeneration were random, but not in the nerve guide repair groups. These findings suggest that PMR, which has previously been documented in rodents, also occurs in primates after certain lesions. The severity of the nerve lesion, including distance and the presence of initial bridging material, may determine whether PMR takes place. In the direct suture and the graft groups, regenerating axons encounter nerve environment immediately after crossing the suture lines. In the nerve guide repair groups, axons must regenerate across an initially empty space, resulting in fewer regenerating motor units eventually contacting muscle.

The statistical analysis to determine the expected number of regenerated motor units after random regeneration assumes that all $\alpha$-motor neurons regenerate and send an axon into the distal stump. It should be stressed that if fewer motor neurons regenerated, the statistically expected number of regenerated motor 

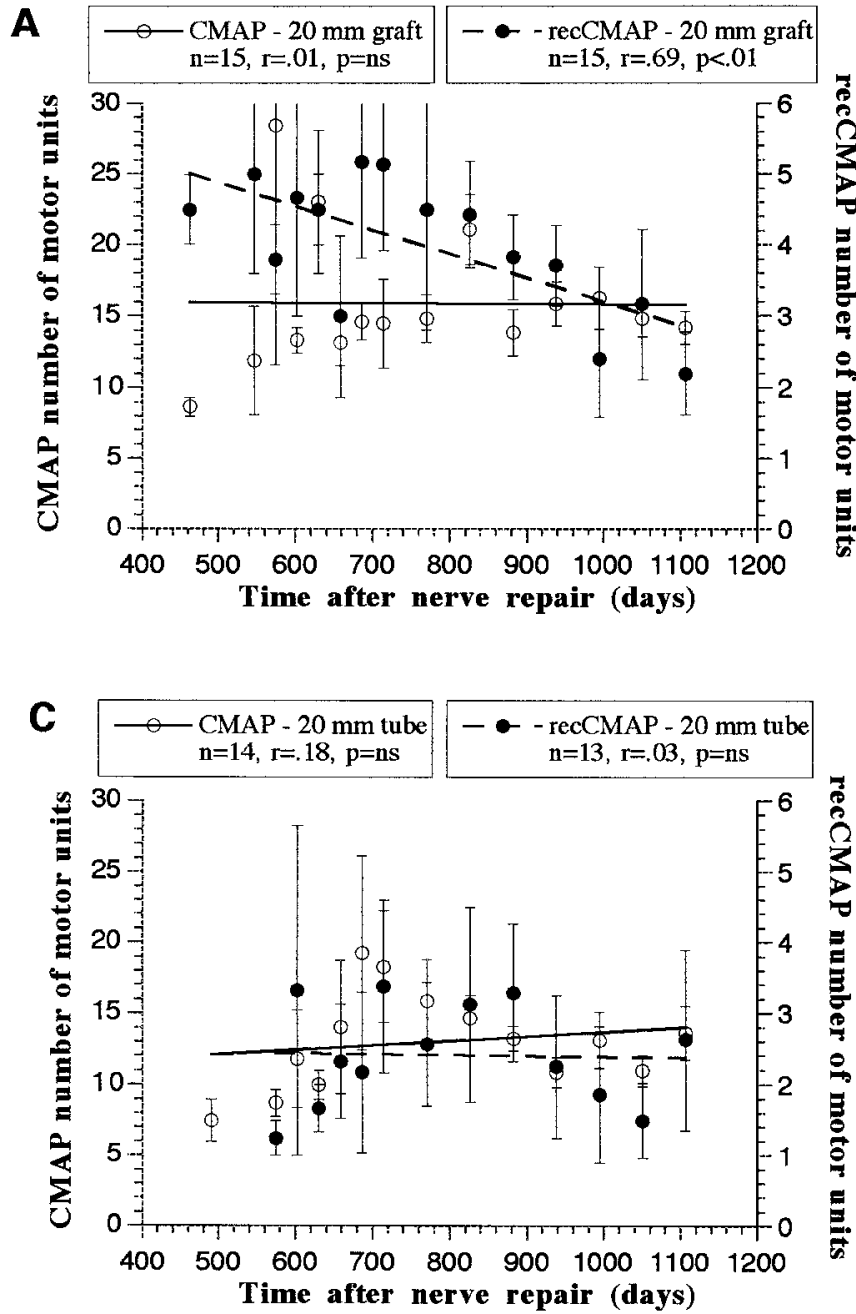
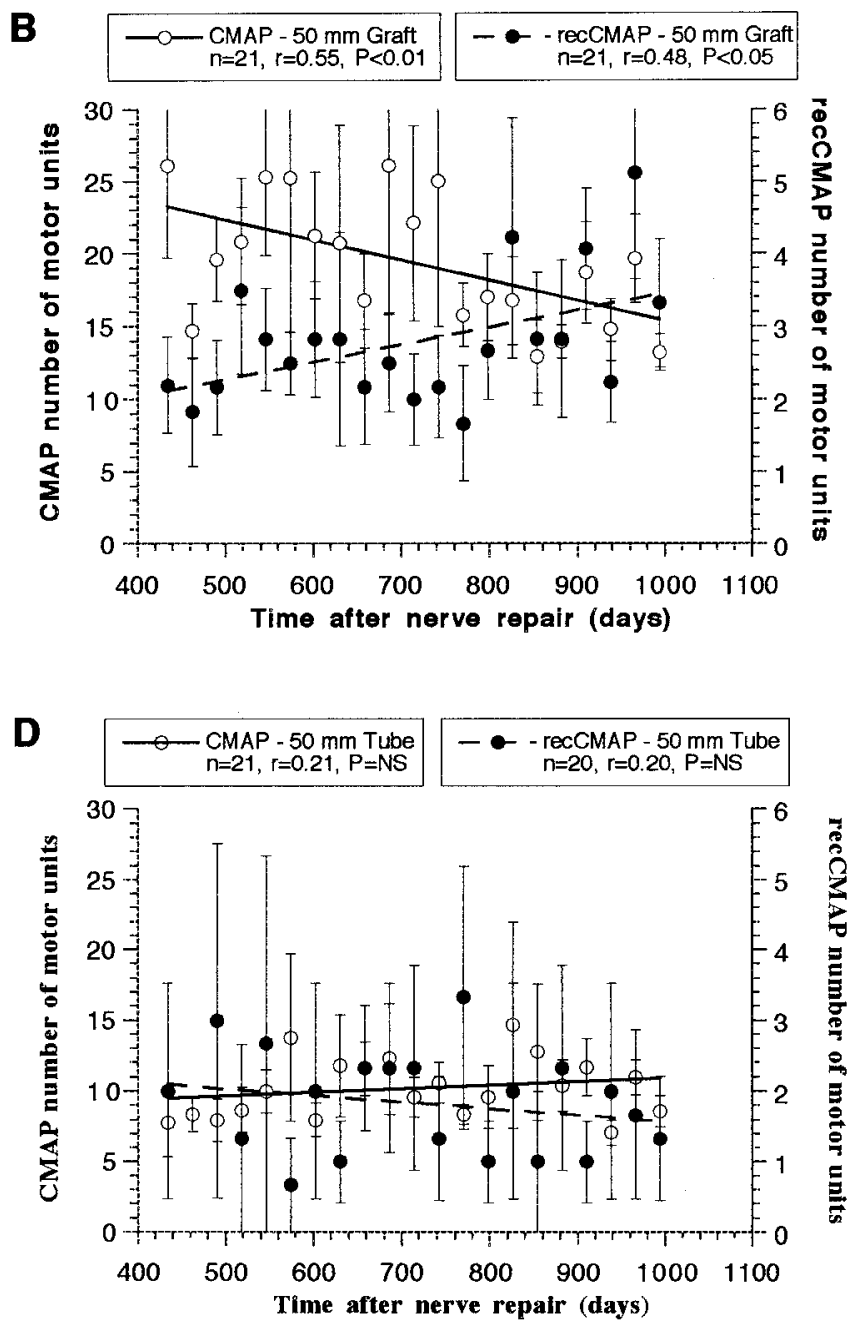

Figure 7. Number of motor units contributing to the CMAP (open circles, left $y$-axis) and the recCMAP ( filled circles, right $y$-axis) with time after nerve repair by sural cable graft $(A, B)$ or collagen nerve guide tube $(C, D)$. Each data point represents the mean ( \pm SEM) of the measurements for that time point. Linear curves were calculated from the least squares method. $n$, Number of time points used to calculate the curve; $r$, correlation between time and number of motor units; $p$, statistical significance of the correlation (two-tailed test); $n s$, nonsignificant.

units would decrease proportionately. Thus our criteria for whether PMR has occurred is conservative.

The average number of motor units in the CMAP in each repair group did not significantly change over the observation period, except for a significant decrease in the $50 \mathrm{~mm}$ graft group. This decline is difficult to fully explain; however, the following possibilities must be considered. We have previously found that a nerve graft in the primate median nerve stimulates significant axonal branching as measured in the distal nerve stump (Archibald et al., 1995). Motor unit dropout could be attributable to increased metabolic stress on the motor neurons as a result of maintaining numerous long axon collaterals within the $50 \mathrm{~mm}$ nerve graft and distal nerve stump as well as innervating muscle. Excessive metabolic stress has been suggested as a reason for the dropout of motor units observed in patients with post-polio syndrome (Dalakas, 1995). The rationale for this happening in the 50 but not the $20 \mathrm{~mm}$ graft groups would be that the greater graft length represents a more severe lesion. Support for this is given by the significantly longer muscle denervation time in the 50 compared with the $20 \mathrm{~mm}$ graft group, as well as a smaller final motor unit size. Alternatively, both the decline in the number of motor units over time and the smaller motor unit size may represent an inability of the long-term denervated muscle and distal nerve sheaths to support the regenerated axons, as has recently been reported after denervation periods of $\geq 6$ months in rodents (Fu and Gordon, 1995b). There would also be a greater time delay before regenerating axons in the $50 \mathrm{~mm}$ graft group begin to grow within basal lamina tubes of the distal nerve, and over time there is a fragmentation of basal lamina tubes in denervated nerve that adversely affects regeneration (Giannini and Dyck, 1990).

All of these unfavorable factors would apply to the nerve guide groups; thus one might expect those groups to have also displayed a reduction in the number of motor units in the CMAP over time. A possible explanation for not detecting such a decline is that the lesions may be so severe that only a few motor neurons display robust enough regeneration to cross the initially empty nerve guide tube in the first place, but once they have innervated muscle they remain relatively stable. Two of five animals in the $50 \mathrm{~mm}$ tube group failed to show any signs of regeneration, highlighting the difficulty of regeneration in the nerve guide groups. 


\section{Aberrant motor axon collaterals in cutaneous nerve}

In all of the regenerated nerves, stimulation of digit II resulted in an aberrant recCMAP. Studies were performed to rule out the possibility of stimulus spread, CNS reflex, or ephaptic transmission. All of the evidence suggests that the most parsimonious explanation for the aberrant recCMAP is that it represents a collateral of an $\alpha$-motor axon, which also innervates muscle; afferent conduction from the digit would occur along the aberrant axon collateral, and after reaching a branching point localized at or distal to the repair site at the wrist, the electrical activity would be conducted efferently along the main axon that innervates muscle.

The conduction velocity along the aberrant motor collateral in the sensory territory initially increased at the same rate as conduction of the sensory fibers giving rise to the CSAP but reached a final level of $\sim 10-15 \mathrm{~m} / \mathrm{sec}$, whereas the CSAP increased to $30-40 \mathrm{~m} / \mathrm{sec}$. These conduction velocities are within the range of myelinated fibers and indicate that both aberrant motor axon collaterals and sensory fibers matured to the point of becoming myelinated. However, the relative conduction velocities also suggest that the aberrant motor axon collaterals only reached a caliber approximately one-third of that of the regenerated sensory fibers in the same digital nerve [(e.g., 3-4 vs 8-9 $\mu \mathrm{m}$, assuming a conversion factor of $4-5 \mathrm{~m} \cdot \mathrm{sec}^{-1} \cdot \mu \mathrm{m}^{-1}$ (Buchthal et al., 1984)]. Thus the digital nerve was capable of supporting large, well myelinated fibers, but the aberrant motor axon collaterals remained quite small.

The absolute refractory period of fibers giving rise to the recCMAP remained 1.5-2.5 times longer than that of the direct motor response, also suggesting that the aberrant fibers had small diameter. The long refractory period could not be used to distinguish whether the diameters of the fibers in both the afferent and the efferent branches differed in size, because the measurement included the entire conduction path. The respective sizes of the axons giving rise to the recCMAP within the aberrant afferent portion (digit to wrist) and the normal efferent motor portion of the pathway (i.e., wrist to muscle) were estimated by comparing the conduction time along both of these pathways. In contrast to the stunted development of the aberrant motor axon collateral within the sensory digital nerve, the conduction velocity of its sibling projecting to muscle was similar to that of the most developed motor axons (i.e., fastest conducting). These refractory period and conduction time results suggest that collaterals from the same motor axon mature to different levels depending on whether they project appropriately to muscle or aberrantly to sensory nerve and suggest greater maturational support when projecting to muscle.

\section{Pruning of aberrant motor collaterals from cutaneous nerve}

Numerous axon collaterals are formed during regeneration (Bray and Aguayo, 1974; Toft et al., 1988). The traditional view has been that once a target (e.g., muscle) is innervated only a single collateral remains (Aitken et al., 1947; Mackinnon et al., 1991; Vrbova et al., 1995; Fu and Gordon, 1997). In our experiments, we would only be able to detect an aberrant motor collateral in the sensory nerve after it acquired functional properties allowing conduction of action potentials and after reinnervation of muscle by its correctly growing sibling axon. A significant decline over time was seen for the number of recCMAPs in the direct suture and $20 \mathrm{~mm}$ graft groups, whereas the number of motor units contributing to the CMAP remained constant. These results suggest a preferential maintenance of the axon collateral that had contacted muscle and a pruning of the sibling collateral in the inappropriate sensory nerve. In all repair groups the motor unit size of the CMAP was significantly greater than the size of the recCMAPs. The consistently smaller size of the recCMAP would suggest that axons with sibling collaterals in the sensory nerve were at a disadvantage in terms of competing during reinnervation of muscle fibers (Colman et al., 1997), even though their conduction properties from wrist to muscle were similar to directly projecting motor axons.

Selective pruning has been suggested to be the anatomical mechanism by which PMR occurs in the rodent femoral nerve (Brushart, 1990), and the present data suggest that a similar mechanism exists in the primate. Interestingly, previous work in the rodent has shown that for pruning to take place the motor neuron must have axon collaterals in both the appropriate muscle branch as well as the inappropriate cutaneous branch. The number of rodent motor neurons that projected exclusively to the inappropriate cutaneous branch did not significantly change over time. This suggests that the active process of pruning requires the ability of the neuron and/or axon to be able to distinguish between the two distinct environments. This process of favoring one axon collateral over all others has been termed "sibling bias," and it has been suggested that it represents the ability of the neuron to compare two distal environments in terms of the level of trophic support (Smalheiser and Crain, 1984; Crutcher and Saffran, 1990). There was no evidence of pruning in the $50 \mathrm{~mm}$ graft group or either of the nerve guide repair groups. In these more severe nerve lesions pruning of aberrant collaterals may not occur or may be markedly delayed. One explanation for the lack of pruning is that there was not enough of a difference between the two distal nerve or target environments to allow sibling bias to result in detectable pruning.

In the $50 \mathrm{~mm}$ graft group there was actually an increase in the number of recCMAP motor units. Both the lack of pruning in this group and the increase in the number of recCMAP motor units may be related. In this group there was a significant postoperative time delay to the first detectable CMAP. This delay suggests that the regenerating axons had a markedly slow advancement; thus aberrant collaterals may have continued to grow into the sensory nerve territory over the period examined. The same rationale would be expected to apply to the nerve guide repair groups, which also had long muscle denervation times, except that these lesions may be so severe that regeneration is significantly blunted overall; thus the number of regenerating motor neurons simply remains quite low.

It is important to stress that because our experimental protocol monitored responses from reinnervated muscle, pruning of aberrant collaterals could occur as well, or even predominantly, during early reinnervation without being detected. Furthermore, a reduction in the number of motor unit potentials in the aberrant motor response may be a conservative estimate of the pruning process, because several collaterals may have been formed, and dropout of an $\alpha$-motor unit potential would require that all conducting collaterals were pruned. Partial pruning would not be discerned as long as even a single collateral could conduct an action potential.

In summary, our results suggest that more motor neurons than expected by random axon regeneration reinnervate muscle after median nerve lesions repaired by direct suture or by nerve grafts of up to $50 \mathrm{~mm}$. In the less severe lesions (i.e., direct suture and $20 \mathrm{~mm}$ graft), pruning of aberrant motor collaterals from sensory 
nerve may be one factor contributing to reinnervation specificity. In all of the repair groups, although aberrant motor axon collaterals remained in sensory nerve territory for long periods, they remained relatively immature, and there was no evidence of innervation of low-threshold mechanoreceptors.

There have been findings of aberrant sensory-motor connections in severe long-term lesions of human nerve (Krarup et al., 1990; Montserrat and Benito, 1990). However, the frequency of such aberrant responses in less severe lesions, their development and changes over time, and the underlying anatomical basis for the response have been unclear. The results of the current experiments suggest that such aberrant connections are quite common. A better understanding of the mechanisms that lead to the pruning of such aberrant collaterals would aid therapeutic intervention strategies designed to increase regeneration specificity and ultimate functional recovery.

\section{REFERENCES}

Aitken JT, Sharman M, Young JZ (1947) Maturation of peripheral nerve fibres with various peripheral connections. J Anat 81:1-22.

Archibald SJ, Shefner J, Krarup C, Madison RD (1995) Monkey median nerve repaired by nerve graft or collagen nerve guide tube. J Neurosci 15:4109-4123.

Bray GM, Aguayo AJ (1974) Reinnervation of peripheral unmyelinated nerves. Fate of axonal sprouts which develop after injury. J Anat 117:517-529.

Brown MC, Hardman V (1987) A reassessment of the accuracy of reinnervation by motorneurons following crushing or freezing of the sciatic or lumbar spinal nerves of rats. Brain 110:695-705.

Brown MC, Hopkins WG (1981) Role of degenerating axon pathways in regeneration of mouse soleus motor axons. J Physiol (Lond) 318:365-373.

Brown WFA (1972) A method for estimating the number of motor units in the thenar muscles and the changes in motor unit count with aging. J Neurol Neurosurg Psychiatry 35:845-852.

Brushart TM (1990) Preferential motor reinnervation: a sequential double-labeling study. Restor Neurol Neurosci 1:281-287.

Brushart TME (1988) Preferential reinnervation of motor nerves by regenerating motor axons. J Neurosci 8:1026-1031.

Brushart TM, Gerber J, Kessens P, Chen Y-G, Royall RM (1998) Contributions of pathway and neuron to preferential motor reinnervation. J Neurosci 18:8674-8681.

Buchthal F, Rosenfalck A, Behse F (1984) Sensory potentials of normal and diseased nerves. In: peripheral neuropathy (Dyck PJ, Thomas PK, Lambert EH, Bunge R, eds), pp 981-1015. Philadelphia: Saunders.

Büngner O (1891) Ueber die regeneration und degenerationvorgange am nerven nach verletzungen. Beitr Pathol Anat 10:321-386.

Cajal RS (1928) Degeneration and regeneration of the nervous system. London: Oxford UP.

Colman H, Nabekura J, Lichtman JW (1997) Alterations in synaptic strength preceding axon withdrawal. Science 275:356-361.

Crutcher KA, Saffran BN (1990) Developmental remodeling of neuronal projections: evidence for trophomorphism? Comments Dev Neurobiol 1:119-141.

Dalakas MC (1995) The post-polio syndrome as an evolved clinical entity. Definition and clinical description. Ann NY Acad Sci 753:68-80.

Fu SY, Gordon T (1995a) Contributing factors to poor functional recovery after delayed nerve repair: Prolonged axotomy. J Neurosci 15:3876-3885.

Fu SY, Gordon T (1995b) Contributing factors to poor functional recovery after delayed nerve repair: prolonged denervation. J Neurosci 15:3886-3895.

Fu SY, Gordon T (1997) The cellular and molecular basis of peripheral nerve regeneration. Mol Neurobiol 14:67-116.

Giannini C, Dyck PJ (1990) The fate of Schwann cell basement mem- branes in permanently transected nerves. J Neuropathol Exp Neurol 49:550-563.

Gutmann E (1945) The reinnervation of muscle by sensory nerve fibres. J Anat 79:1-8.

Gutmann E, Sanders FK (1943) Recovery of fibre numbers and diameters in the regeneration of peripheral nerves. J Physiol (Lond) 101:489-518.

Henneman E (1974) Peripheral mechanisms involved in the control of muscle. In: Medical physiology (Mountcastle VB, ed), pp 617-635. St. Louis: Mosby.

Holmes W, Young JZ (1942) Nerve regeneration after immediate and delayed suture. J Anat 77:63-96.

Ide C (1983) Schwann cell basal lamina and nerve regeneration. Brain Res 288:61-75.

Krarup C, Upton J, Creager MA (1990) Nerve regeneration and reinnervation after limb amputation and replantation: clinical and physiological findings. Muscle Nerve 13:291-304.

Lee MT, Farel PB (1988) Guidance of regenerating motor axons in larval and juvenile bullfrogs. J Neurosci 8:2430-2437.

Li ST, Archibald SJ, Krarup C, Madison R (1992) Peripheral nerve repair with collagen conduits. Clin Mater 9:195-200.

Mackinnon SE, Dellon L, O'brien J (1991) Changes in nerve fibre numbers distal to a nerve repair in the rat sciatic nerve model. Muscle Nerve 14:1116-1122.

Madison RD, Archibald SJ (1996) Potential value of synthetic tubes for nerve repair. In: Neurosurgery (Wilkins RH, Rengachary SS, eds), pp 3193-3197. New York: McGraw-Hill.

Madison RD, Archibald SJ, Brushart TM (1996) Reinnervation accuracy of the rat femoral nerve by motor and sensory neurons. J Neurosci 16:5698-5703.

McLeod JG, Wray SE (1967) Conduction velocity and fibre diameter of the median and ulnar nerves of the baboon. J Neurol Neurosurg Psychiatry 30:240-247.

Montserrat L, Benito M (1990) Motor reflex responses elicited by cutaneous stimulation in the regenerating nerve of man: axon reflex or ephaptic response? Muscle Nerve 13:501-507.

Parry GJ, Brown MJ (1982) Selective fiber vulnerability in acute ischemic neuropathy. Ann Neurol 11:147-154.

Rasminsky M (1980) Ephaptic transmission between single nerve fibres in the spinal nerve roots of dystrophic mice. J Physiol (Lond) 305:151-169.

Scherer SS, Easter Jr SS (1984) Degenerative and regenerative changes in the trochlear nerve of goldfish. J Neurocytol 13:519-565.

Scott JJA (1987) The reinnervation of cat muscle spindles by skeletofusimotor axons. Brain Res 401:152-154.

Sica REQ, McComas AJ, Upton ARM, Longmire D (1974) Motor unit estimation in small muscles of the hand. J Neurol Neurosurg Psychiatry 37:55-67.

Smalheiser NR, Crain SM (1984) The possible role of "sibling neurite bias" in the coordination of neurite extension, branching, and survival. J Neurobiol 15:517-529.

Swett JE, Wikholm RP, Blanks RHI, Swett AL, Conley LC (1986) Motor neurons of the rat sciatic nerve. Exp Neurol 93:227-252.

Toft PB, Fugleholm K, Schmalbruch H (1988) Axonal branching following crush lesions of peripheral nerves of rats. Muscle Nerve 11:880-889.

Vrbova G, Gordon T, Jones R (1995) Nerve regeneration and muscle reinnervation. In: Nerve-muscle interaction (Vrbova G, Gordon T, Jones R, eds), pp 197-235. London: Chapman and Hall.

Weiss P, Edds MV (1945) Sensory-motor nerve crosses in the rat. J Neurophysiol 30:173-193.

Wray SE (1969) Innervation ratios for large and small limb muscles in the baboon. Journal Comp Neurol 137:227-250.

Zalewski AA (1970) Effects of reinnervation on denervated skeletal muscle by axons of motor, sensory, and sympathetic neurons. Am J Physiol 219:1675-1679.

Zar JH (1984) The binomial distribution. In: Biostatistical analysis, pp 369-405. Englewood Cliffs, NJ: Prentice Hall. 